\title{
CONDITION ASSESSMENT OF ROADWAY BRIDGES: FROM PERFORMANCE PARAMETERS TO PERFORMANCE GOALS
}

\section{MARIA PINA LIMONGELLI ${ }^{*}$, ELENI CHATZI ${ }^{2}$, ANDREJ ANŽLIN ${ }^{3}$ \\ ${ }^{1}$ Dept of Architecture, Built Environment and Construction Engineering, Politecnico di Milano, Milano, Italy \\ ${ }^{2}$ Dept of Civil, Environmental and Geomatic Engineering, ETH Zurich, Zurich, Switzerland ${ }^{3}$ Dept of Structures, Slovenian National Building and Civil Engineering Institute, Ljubljana, Slovenia}

Received 24 November 2017, accepted 23 August 2018

\begin{abstract}
Deterioration of bridges due to ageing and higher demands, induced by increased traffic load, require the development of effective maintenance policies and intervention strategies. Such concern should be aimed at ensuring the required levels of safety, while optimally managing the limited economic resources. This approach requires a transversal advance; from the element level, through the system level, all the way to the network level. At the same time intervention prioritisation based on the importance of the system (bridge) inside the network (e.g. highway), or of the single structural element inside the bridge is dependent. The first step in bridge condition assessment is the verification of safety and reliability requirements that is carried out using the traditional prescriptive (deterministic) approach or the current performancebased (probabilistic) approach. A critical issue for efficient management of infrastructures lies in the available knowledge on condition and performance of bridge asset. This information is obtained using a collection of significant Performance Parameters at one or more of the three levels (element, system, and network). Traditional techniques for estimation of Performance Parameters rely
\end{abstract}

\footnotetext{
* Corresponding author. E-mail: mariagiuseppina.limongelli@polimi.it 
on already established visual inspection. However, a more reliable description of the system performance is obtained through Non-Destructive Testing and Structural Health Monitoring. Condition assessment essentially pertains to the check of compliance with Performance Goals and requires the definition and computation of Performance Indicators. They are calculated directly from Performance Parameters or from physical models calibrated using the Performance Parameters collected on the structure. Paper overviews the steps to bridge condition assessment regarding safety and reliability.

Keywords: condition assessment, Performance Goals (PGs), Performance Indicators (PIs), Performance Parameters (PPs), reliability, safety.

\section{Introduction}

Most of the roadway bridges, built before the adoption of modern principles of sustainable planning and seismic design, are approaching their design lifetime. The volume and loading of the heavy freight vehicles, which they are carrying, are considerably larger than anticipated at the time of their construction. In most cases, these bridges are structurally deficient and degraded due to the ageing effects and inadequately maintained (Figure 1). Reliable assessment of their safety to seismic and increased operational loads is therefore required before deciding on their optimal management.

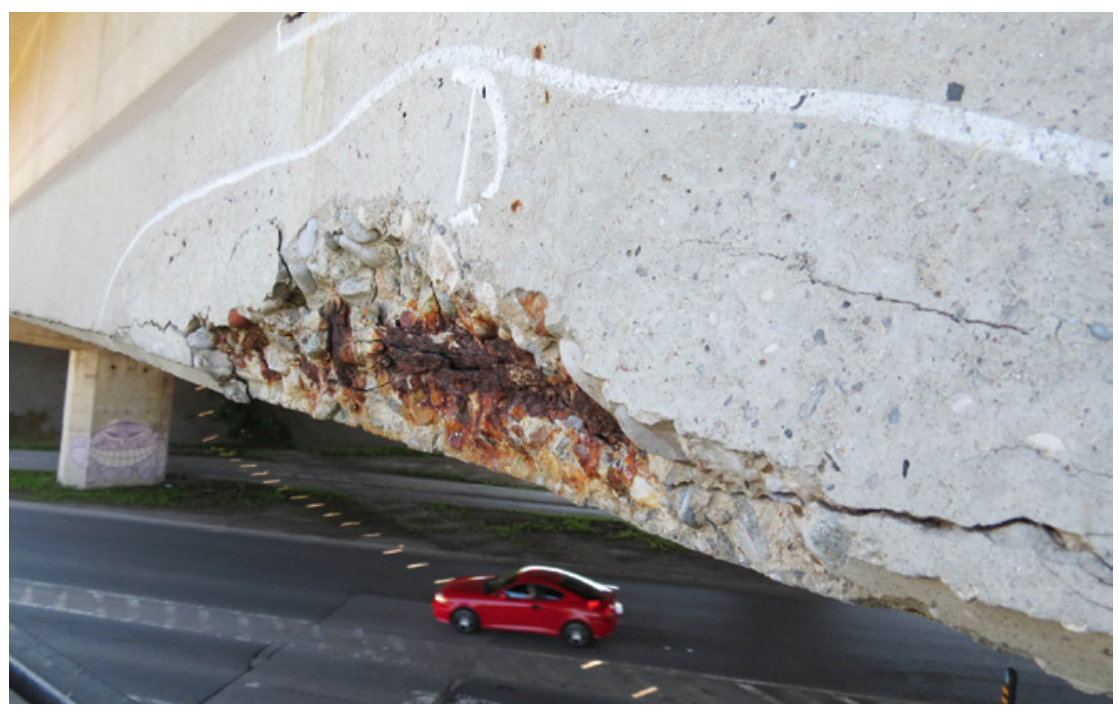

Figure 1. Delaminated concrete cover due to the corrosion of the reinforcement on a roadway bridge 


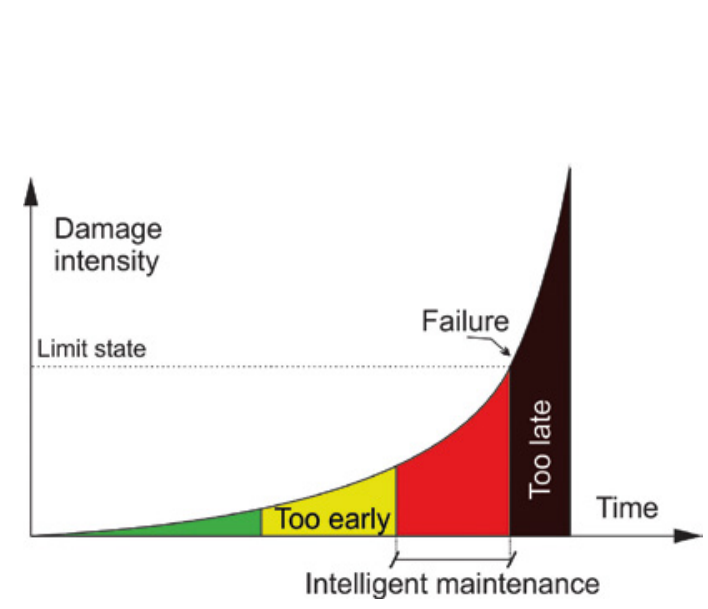

Figure 2. The concept of intelligent maintenance

Efficient maintenance of the infrastructure is a process that exerts excellent economic pressure on their owners and managers. In deciding on further remedial or rehabilitation measures, it is, therefore, necessary to look for solutions that follow the concept of intelligent maintenance. Such actions are in this way optimally scheduled along the life cycle of the structure (Figure 2). A methodological framework is therefore needed for assessment of the resilience of such structures that allows for consideration of the most realistic state of damage to the structure at a given time. The accuracy of such assessment depends on the type of the mathematical model, as well as on the availability of the data upon, which the assessment was based, i.e. the availability of the Performance Parameters (PPs). One of the candidate approaches, allowing for the inclusion of a broad spectrum of information, is the probabilistic conditional assessment that is mentioned in this paper. Please note that the focus is herein given on the Performance Goals (PGs) of safety and reliability.

\section{Inspection and monitoring: a collection of Performance Parameters}

Performance parameters are information (measure or expert opinion) collected using visual inspections, off-site tests on materials (Destructive Tests), on-site investigation using the Non-Destructive Tests (NDT), or Structural Health Monitoring (SHM) systems. These parameters support the procedures for bridge assessment, and their collection has to be in line with the pre-defined Key Performance Indicators (KPIs). 
An obvious bottleneck in bridge assessment lies in the treatment of qualitative information. Such data, which is usually retrieved through periodical visual inspections, strongly rely on expert subjective opinion for assessment of structural condition. An objective assessment ought to be instead put in place that in turn heavily relies on the availability, ease of implementation and resolution of monitoring and inspection methods.

Current inspection procedures adopted in Europe for collecting structural information are classified into four main categories. They can be listed at an increasing level of accuracy in the quantification of the PPs, along with an accompanying increase in costs as:

- Visual inspections;

- Destructive Testing;

- Non-Destructive Testing;

- Structural Health Monitoring techniques.

\subsection{Visual inspections}

Visual inspection forms the "de-facto" tool of structural assessment in both Europe and the rest of the world. In only a few European countries an official procedure is offered for condition assessment of bridges, while official regulations on the inspections needed for the collection of PPs are not established.

European Bridge condition assessment is for the most part traditionally based on a rating system. A specific number of condition levels corresponding to different levels of degradation, usually from zero (no damage) to the maximum level. The latter corresponds to defects that jeopardize the safety and thus require immediate intervention and limitation or shutdown of traffic. The rating is assigned based on the results of visual inspections that are regularly carried out by technicians. Their goal is mainly related to the detection of local damage parameters, e.g. cracks, concrete spalling or loss, delamination, steel corrosion. Based on the extent of such damages a rating is assigned based on the scale above.

The main shortcomings related to the information retrieved from visual inspection are summarized as:

- they form local information related to the single structural section or structural element not allowing the condition rating of the structure as a whole;

- they are more often qualitative and not quantitative information, leaving room for subjective interpretations of experienced bridge engineers, rather than leading to objective evaluation of the structural conditions.

Based on their frequency, as hereinafter reported in the Strauss, \& Mandić-Ivanković (2016) WG1 report, such inspections are classified into four main categories: 
- routine inspections, e.g. yearly basis;

- simple checks, e.g. three years after every main inspection;

- in-depth examinations or main inspections, e.g. every six years;

- special inspections, following exceptional occurrences or incidents.

\subsection{Destructive Testing}

Destructive Testing (DT) renders quantitative information on material parameters (e.g. the strength of materials and elastic modulus) and structural integrity (corrosion ingress). It is carried out by extracting samples from the structure and follow-up laboratory test. The drawback is the invasive character of these type of tests and the local character of the information they provide. Such data also depends on the location of the sample used for the laboratory test.

\subsection{Non-Destructive Testing}

Non-Destructive Testing (NDT) methods aim in providing information on the structural condition, without harming the structure itself, i.e. in a non-invasive manner since they do not require samples of material taken from the structure. A wide variety of non-destructive technologies are available for bridge structure, e.g. Ground Penetrating Radar, Acoustic emission, Thermography methods, Magnetic flux leakage. They provide local information on the conditions of both individual structural elements (e.g. rebar, post-tensioning) and non-structural elements (such as the location of voids, pipes, pavement thickness). Detection of zones with increased chloride contents and moisture is also possible and provides a warning on deterioration through corrosion. This list is incomplete, and the interested reader is referred to the work of Ayswarya, Johnson, Chaithanya, Prasad, Krishnan, \& Nair (2016) for further information. NonDestructive Testings offer a more rigorous quantitative characterisation of the structure concerning visual inspections. Their shortcoming is related to the local character of the information they provide, which requires expensive testing campaigns, to achieve a global description of the structure.

In addition, static load tests using loaded trucks are considered to be a particular family of NDTs and constitute one of the standard means of structural testing for determining structural capacity. Shortcomings of these tests are related to the prohibitive costs, and rather a long interval within the operation of the bridge needs to be suspended. Additionally, the limitations related to the maximum size of the truck as well as the maximum load that is allowed to carry are met. 


\subsection{Structural Health Monitoring}

The state-of-the-art in retrieving PPs relies on the use of monitoring systems, namely sensor network deployed on the structure, able to record the structural response to operational loads, ambient vibrations or seismic excitations. These records are applied in the static or dynamic sense, allowing assessing or characterising the system, using inverse or system identification techniques. The significant advantage concerning the previous technique is that they allow computing global PPs, thus rendering an objective evaluation of the structural condition of the bridge as a whole.

The use of monitoring systems for bridge structures is becoming more and more established (Wenzel, 2008). The implementation of such systems is classified depending on the duration of the instrumentation. The first main category is short or mid-term monitoring, typically continuing from up to few days to few weeks of instrumentation, respectively. The second category is long-term monitoring continuing from a few months to a few years, and perhaps throughout the lifespan of the structure (Glišić, Posenato, \& Inaudi, 2007). A noteworthy example of short-term monitoring for condition assessment and immediate decisionmaking processes is the Non-Destructive Dynamic Field Testing (from vibration response data) conducted in three Cincinnati bridges for the rating of those specimens (Aktan, Chuntavan, Lee, \& Farhey, 1994). The testing methods utilised in that case included impact tests as well as proof-load level truckload tests.

The main issue in damage identification and condition assessment through monitoring data is the fact that environmental effects also play a significant role in the properties of the system. In this sense, long-term monitoring (from cradle-to-grave) is advisable for continually tracking the evolution of the system properties under environmental, operational and deterioration effects. Long-term monitoring systems have already been implemented on some bridges in Europe (Casciati, 2003), the United States (Pines, \& Aktan, 2002), and elsewhere. An example of a state-ofthe-art implementation is the long-term monitoring system deployed on the Tsing Ma bridge in Hong Kong (Chung, Liu, Guan, Chan, Chan, \& Tam, 2003), involving a network of more than 350 sensor channels including Global Positioning System (GPS) and Fibre Bragg Grating (FBG) sensors. A further pioneering monitoring initiative is the one initiated by the Californian Dept of Transportation (Caltrans, 2006) and the California Strong Motion Instrumentation Program (CSMIP) for instrumenting Caltrans bridges throughout the state, recording their response during earthquakes. This data is assimilated with an additional data stream from further infrastructure components for identifying the areas of highest potential damage. This blend of information is afterword used 
by the Office of Emergency Services and other emergency response personnel in the event of a damaging earthquake.

Although still relatively rare, such schemes are becoming more and more available. As the necessary technology becomes increasingly cheaper and software systems become more and more spread, such schemes are envisioned as the future of monitoring. Eventually, traditional assessment methods, such as visual inspection, will be accompanied with such assessment systems.

\section{Condition assessment: from Performance Parameters to Performance Indicators}

The information (PPs) stemming from monitoring and inspection is used in three major ways:

- for computation of the Performance Indicators (PIs) (also termed Condition Indices) and direct check of PGs (thresholds), where a direct comparison is feasible (e.g. crack length, load and strain thresholds); Performance Indicators coincides with PPs (for example in the case of crack length where the existence of a crack is by itself an indication of damage) or is computed as functions of the PPs (for example variation of stiffness concerning the undamaged condition). Usually, the direct computation of PIs from PPs is limited to assessment at the element level;

- for the computation of system or network PIs able to describe the state of the asset with different degrees of refinement (depending on the amount of available information); the latter is particularly relevant in the case of information obtained from monitoring systems (Limongelli, Chatzi, Döhler, Lombaert, \& Reynders, 2015), for instance, about response under ambient or forced vibrations;

- for the updating and calibration of structural models, to reflect the actual state of the bridge system; recalculation of the relevant indicators is then possible with increased confidence; this approach is adopted for example in modal parameters (frequencies, damping factors, and modal shapes) retrieved from dynamic tests or continuous monitoring systems.

\subsection{From Element Performance Indicators to System Performance Indicators}

When PIs need to be computed at the structural level, the importance of every single element for the bridge functionality has to be appropriately taken into account (Strauss, \& Mandić-Ivanković, 2016). Based on the 
computational approach used, the methods for developing PIs (also defined as Bridge Condition Indices) from element PIs is herein grouped into the following four approaches (Chase, Adu-Gyamfi, Aktan, \& Minaie, 2016):

- the weighted averaging approach that estimates the condition of the whole structure. It uses the combinations of condition ratings of all individual bridge elements weighted by their significance or contribution to the structural integrity of the bridge. This approach is common in systems, which rely on element-level inspection data. Bride Condition Index (BCI) used in Australia (Bridge Condition Number - BCN), the United Kingdom (BCI), South Africa (BCI), and Austria (BCI);

- in the worst conditioned component approach, the BCI is approximated to the condition index of the component in the worst condition. The German and Japanese BCIs are the examples of such an approach, which is common in systems that carry out inspections on key bridge components;

- qualitative methods do not report the condition of the bridge on a numerical scale. They describe either structure as "Poor", "Fair", or "Good", based on the condition and importance of the elements under investigation. This type of methods is used in the United States (Washington, Florida, and other States);

- ratio-based methods assign a $\mathrm{BCI}$ or $\mathrm{BCN}$ based on the ratio of the PPs in the current condition concerning the value of the same PP measured in the structure when it was first built.

\subsection{From System Performance Indicators to Network Performance Indicators}

Each element of the network has to be assessed according to codes enforced in the different European countries or at the European level (e.g. Eurocodes). The assessment has to be in accordance to prescribed standards and guidelines to comply with safety requirements.

However, for safety and reliability assessment a competing requirement exists among codes on one side, requiring the structure to be safe under design actions, and the limited resources on the other side. Owners and concessionaires in charge of bridge maintenance are thus led to establishing a hierarchy of interventions. The importance of the bridge in the network to prioritise investments is therefore accounted.

In this respect, it is of strategic importance to define PIs at the network level. In such a way the choice among maintenance options for bridges, which do not comply with code safety requirements, as well as the prioritisation of interventions in bridges of the network is guided. Therefore, the network PIs are usually linked to costs related 
to the maintenance activity and indirect costs caused by maintenance activities. These PIs can also be borne by the society, such as user delay (availability) and environmental impacts, and with aspects related to traffic safety (Stipanovic, Chatzi, Limongelli, M., ..., \& Ademovic, 2017).

For each of these aspects, a KPI is defined (Availability, Economy, Environment, and Traffic Safety) and the proper PPs are computed. Herein attention is focused on PIs and PGs related to the KPIs of Safety and Reliability thus attention is focused on Element and System PIs.

\section{Condition assessment: prescriptive vs probabilistic approach and related Performance Goals}

The computation of PIs from PPs further depends on the approach for Condition assessment which dictates the performance goals to be met by the bridge.

The traditional prescriptive approach requires the computation of PIs regarding parameters representatives of the structural behaviour at the Service or Ultimate Limit State to be checked against predefined thresholds. For example, in many seismic-prone areas throughout Europe bridges have been designed according to old codes. In those codes, the PG is defined as the achievement of a given demand to capacity ratio, in the context of preservation of life and safety of the users. Other PGs related to the limitation of damage, maintenance of functions or provision for easy repairs were not at all considered in this traditional approach.

The assessment is carried out based on structural reliability methods, while Capacity and Demand are expressed regarding Resistance and Actions on the structure. The Actions on the structure are fixed by the codes usually through a response spectrum.

These methods do not explicitly address the probability of occurrence of the earthquake or the costs of the consequences. These are implicitly taken into account in the definition of the actions on the structure (response spectrum) and of the behaviour factor (used to reduce the actions on the structure based on its dissipation capacity).

The Performance Goal of the modern performance-based approach in seismic design lies in the achievement of a certain level of performance while accounting for related consequences. In contrast to the traditional methods, performance-based assessment is carried out, making decisions based on the desired level of performance.

Risk methods are applied considering exposure and hazard instead of actions as well as vulnerability instead of capacity and consequences.
Condition

Assessment

of Roadway Bridges:

from Performance

Parameters

to Performance

Goals 
In a prescriptive design, such methods are not explicitly accounted. In the definition of the seismic hazard, the probability of occurrence of the seismic event is explicitly taken into account and related to vulnerability through the fragility curves. They describe the probability of a considered state of the system (no damage, low damage, high damage, collapse) as a function of the seismic hazard level. The fragility curves are developed at the component, structure or network level. Great efforts are currently devoted to computation at the later level. The fragility curves are then used to obtain the probabilities of different damage states (levels) and the probability of failure (or the reliability index $\beta$ ). The risk is computed by considering the costs of different consequences, e.g. casualties or monetary losses, related to the considered performance level. Decisions about possible interventions of the structure are then made based on the costs of different performance levels.

Consideration of monitoring methods relying on strain measurements, vibration, water content or site testing allows for a much more accurate calculation of the reliability. The information is, therefore, be considered stochastically in the analysis using the maximum likelihood estimation of distribution parameters or Bayesian statistical updating of the previously calculated statistical models. If the risk is still found to be unacceptable, repair and remediation strategies need to be considered in the reliability domain to optimise their performance.

Target values of the reliability $\beta$ are given in EN 1990:2002 Basis of Structural Design for the design of structures but not for assessment of existing ones. Since 2016, the International Federation for Structural Concrete $(f i b)$ has established a large international Committee for developing Model Code 2020 for Concrete Structures combining both for design and assessment of structures. At the European level, significant research efforts focus on the development of reliability assessment frameworks, such as in Europe Union project DESTination Rail. Often, adoption of a probabilistic approach accounting for testing and monitoring information reveals significantly higher capacity than the one calculated using subjective visual inspection information. Furthermore, the probabilistic approach when applied alongside the exact whole life cycle cost offers a more reliable calculation of risk by quantifying and possibly reducing associated uncertainties.

\section{Conclusions}

Due to the increasingly intensified and dynamic loading conditions, as well as the underlying ageing and deterioration processes, it is imperative to come up with adequate techniques for optimally managing 
bridge infrastructure. Additionally, the safety and capacity of the existing transport infrastructure are eventually reduced due to the constant increase of the average age and consistent growth of freight and passenger traffic. Consequently, particularly near transport nodes, the number of congestions and bottlenecks is increasing. Shortly, for the needs of sustainable mobility, an assessment of the resilience of the existing infrastructure will comprise a mandatory and standardised check. Such an approach, although unusual for bridges will result in a significant engineering and investment challenge.

To conclude, it is widely advisable, that the selection of remediation and rehabilitation measures under various risks and uncertainties is:

- based on the comprehensive conditional assessment of the structure;

- supported by monitoring information and treated using a probabilistic approach.

In this way, the selection of the most effective measure to improve the condition of the structure and to ensure sufficient structural safety will be ensured.

\section{REFERENCES}

Aktan, A. E., Chuntavan, C., Lee, K. L., \& Farhey, D. N. (1994). Nondestructive testing and identification for bridge rating, phase 2: steel-stringer bridges.

Ayswarya, K.S., Johnson, A.M., Chaithanya, Prasad, D., Krishnan, R.D., \& Nair, R.N.J. (2016). Evaluation of Bridge Performance Using Non-Destructive Testing - a Review, International Advanced Research Journal in Science, Engineering and Technology 5(1): 5-18. https://doi.org/10.17148/IARJSET

Caltrans (2006). Seismic Design Criteria 1.4, California Dept of Transportation, California.

Casciati, F. (2003). An overview of structural health monitoring expertise within the European Union. Structural health monitoring and intelligent infrastructure, 31-37.

Chase, S. B., Adu-Gyamfi, Y., Aktan, A. E., \& Minaie, E. (2016). Synthesis of national and international methodologies used for bridge health indices (No. FHWA-HRT-15-081). United States. Federal Highway Administration.

Chung, W. H., Liu, S. Y., Guan, B. O., Chan, T. L., Chan, T. H., \& Tam, H. Y. (2003, September). Structural monitoring of Tsing Ma Bridge using fiber Bragg grating sensors. In Optoelectronics, Proceedings of the Sixth Chinese Symposium (pp. 144-146). IEEE. https://doi.org/10.1109/COS.2003.1278186 EN 1990:2002 Basis of Structural Design.

Glišić, B., Posenato, D., \& Inaudi, D. (2007, April). Integrity monitoring of an old steel bridge using fiber optic distributed sensors based on Brillouin scattering. In Nondestructive Characterization for Composite Materials, 
Aerospace Engineering, Civil Infrastructure, and Homeland Security 2007 (Vol. 6531, p. 65310P). International Society for Optics and Photonics. https://doi.org/10.1117/12.716055

Limongelli, M. P., Chatzi, E., Döhler, M., Lombaert, G., \& Reynders, E. (2016, July). Towards extraction of vibration-based damage indicators. In EWSHM-8th European Workshop on Structural Health Monitoring.

Pines, D., \& Aktan, A. E. (2002). Status of structural health monitoring of longspan bridges in the United States. Progress in Structural Engineering and materials, 4(4), 372-380. https://doi.org/10.1002/pse.129

Stipanovic, I., Chatzi, E., Limongelli, M., ..., \& Ademovic, N. (2017). WG2 technical report: Performance goals for roadway bridges of COST Action TU 1406. $82 \mathrm{p}$.

Strauss, A., \& Mandić-Ivanković, A. (2016). WG1 technical report: Performance indicators for roadway bridges of COST Action $1406.40 \mathrm{p}$.

Wenzel, H. (2008). Health monitoring of bridges. John Wiley \& Sons. 